Original Research Paper

\title{
Swarm Optimization Techniques for Segmenting Gel Electrophoresis Images
}

\author{
Sara Ibrahim Ibrahim, Mohamed Abd Allah Makhlouf, Ghada.S. El-Tawel and M.E. Wahed \\ Department of Information System, Suez Canal University, Ismailia, Egypt
}

\author{
Article history \\ Received: 22-07-2015 \\ Revised: 05-10-2015 \\ Accepted: 18-06-2016 \\ Corresponding Author: \\ Sara Ibrahim Ibrahim \\ Department of Information \\ System, Suez Canal University, \\ Ismailia, Egypt \\ Email: gana2491990ex@gmail.com
}

\begin{abstract}
Gel Electrophoresis (GE) are discussed as the main tool to dissociate DNA sequences. It helps in analyzing the genome such that each image resulting from it consists of lanes that include several bands. Image segmentation plays the foremost role in image processing. It helps in producing accurate results in medical diagnosis. Image segmentation works by dividing an image into regions that cover the full image. Image segmentation methods can be implemented, but still have certain defects that cannot produce accurate results. On the other hand, Swarm Optimization methods produce results with high efficiency in image segmentation. In this study, swarm optimization techniques for image segmentation are proposed. The proposed technique depends on applying different segmentation methods as Fuzzy C-Means (FCM) and Particle Swarm Optimization (PSO) is an extensively used in computer science considered a simple and easy algorithm to implement. It also depends on swarm intelligence. PSO useful in image segmentation because the result is more exact and efficient. Furthermore, Darwinian PSO (DPSO) and Fractional Order Darwinian PSO (FODPSO) produced precise results. The efficiency of the proposed approach is compared with other by computing image quality measurement parameters like Peak Signal to Noise Ratio (PSNR), Mean Square Error (MSE) and others. The proposed technique, especially FODPSO produces more accurate results to segment GE image.
\end{abstract}

Keywords: DNA, Electrophoresis Gel, Image Denoising, Image Preprocessing, Image Segmentation, Clustering, FCM, PSO, DPSO and FODPSO

\section{Introduction}

Deoxyribonucleic Acid (DNA) is the backbone of all living organisms (Zhu et al., 2011). DNA comprises of double strands of sugar, connected together by nucleotide bases. It also has four bases; Adenine (A), Cytosine (C), Guanine (G) and Thymine (T). The varieties of DNA of different living beings in the whole world depend on the variety in the length of the helix and its order. It is very easy to determine a sequence of bases if you know the sequence of bases on one side of the double strand. Since the two strands are compliments like (A) is complemented with (T) and $(\mathrm{C})$ is complemented with $(\mathrm{G})$. Figure 1 displays two strands of DNA.

The DNA sequence is the process of specifying the accurate rank of the four bases of DNA or RNA. It's helpful for human recognition, which is used in genetic testing and fingerprint, is unique for every person. There are several techniques for DNA sequence like MaxamGilbert sequencing in (1977) by Allan Maxamand Walter Gilbert and Sanger Sequencing, the popular technique for DNA sequence, by Fredrick Sangerat (1977) (França et al., 2002). These techniques depend on a number of bases. If DNA sequences are above 1000 base pairs, then use the Shotgun sequencing and electrophoresis.

Electrophoresis, discovered by Fred Sanger, is the main tool for DNA sequencing. It divides a molecule in to several pieces of different sizes by restriction enzymes (Lee et al., 2011). Gel electrophoresis is used to divide DNA pieces according to size and an electric field. If the DNA is negatively charged, it will move towards the electrode of opposite charge (Fig. 2). 


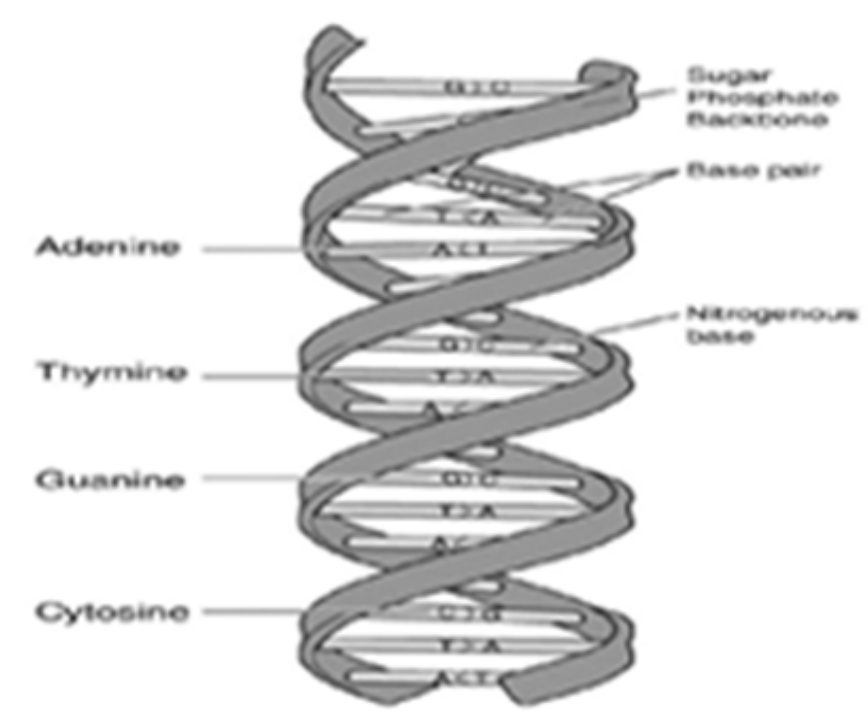

Fig. 1. DNA double-strands (Nelson et al., 2008)

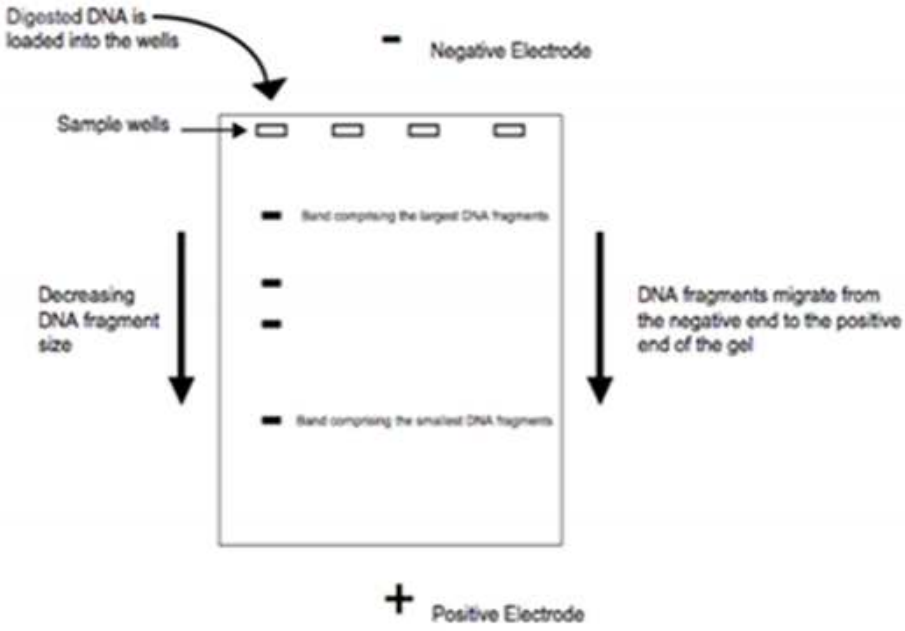

Fig. 2. Migration of DNA fragments

There are several types of gel the selection depending on the sizes of basis. Agarose gel divides great nucleic acids, to short nucleic acids and proteins using Acrylamide gel (Akhter et al., 2008). The result of this method is a single image that includes multiple lanes which are vertical. There are several horizontal bands for every lane. Image resulted from Gel Electrophoresis still the main way to cope with a DNA sequence. The principal defect of the Gel Electrophoresis image is suffering from multiple noises that lead to a reduction in the image quality, so image processing is very useful in this area. In this study, image processing is composed of three levels: Image preprocessing, image segmentation and result evaluations. Image Preprocessing is the principal level in this approach. It consists of image enhancement techniques as applied filtering. It is also useful to display the clearest image Taher et al. (2013). Image segmentation is the next step in this approach. It is the method that separates an image into regions that cover the image. It is important to apply segmentation methods in the gel electrophoresis image to detect all bands and lanes and to use the resulted image to identify diseases such as breast cancer. Also, it is important because it's the second step after removing noise from the image. SWARM comes from a moving group of birds searching for food in a search space. They have not known the best area where food is in therefore, if any bird detect the destination, then all of combination will move toward it.

The core problem of multiple segmentation methods is producing defected results such as the result is not accurate enough to segment gel electrophoresis image. 
So, the aim of this paper is to solve this problem by applying multiple Swarm Optimization techniques. Since they produce more accurate and high efficient results for medical image segmentation. The proposed segmentation method applies Fuzzy C-Means (FCM) and Particle Swarm Optimization (PSO). PSO is a stochastic optimization algorithm that depends on swarm intelligence and Darwinian PSO (DPSO) to solve the main problem that faced PSO and Fractional order Darwinian PSO (FODPSO) more popular techniques in image clustering and image segmentation and compute results by using image quality measurements. Then compare the preceding techniques to show the best.

The main contribution of this paper is to produce pure segmented images with clear bands and lanes without losing the features of images because they real images. Then classify the resulted image to detect breast cancer disease.

The rest of this paper divided into four sections: Section Two contains the methods that have been applied to image segmentation. It is divided into two subsections FCM and SWARM Optimization. SWARM Optimization is divided into three Sub subsections: PSO, DPSO and FODPSO. Section three includes two Subsections which state steps for preprocessing and illustrate a block diagram of the proposed segmentation technique. Section four includes three Subsections in Subsection Data Sets presents the image data sets that have been tested. In subsection Performance Evaluation shows some figures after applying segmentation techniques and performing comparisons between results using specific parameters. In subsection comparison with other technique displays their results in tables. After performing comparisons between results using specific, image error measurements to obtain the best result. Section Five explores the paper summary and its conclusion.

\section{Related Work}

Many researchers had applied several segmentation techniques that performed on gel electrophoresis images to get more accurate and efficient output (Talukder, 2011).

Noor et al. (2011) proposed multilevel thresholding Otsu method based on Particle Swarm Optimization to segment Gel Electrophoresis image for DNA. After experimenting with this technique, efficient results for segmenting all bands in the gel electrophoresis image were produced. The disadvantage of this technique is that it did not segment all lanes.

Sengar et al. (2012) applied watershed method using wavelet transform to segment 2D Gel Electrophoresis image as protein spots. The advantage of this technique is the fact that it applies a single threshold factor. This technique is helpful to segment spots, but there are some missing spots after segmentation.
Ahmad et al. (2013) proposed segmentation of the Gel Electrophoresis image as DNA by PSO technique with Kapur multilevel thresholding. The aim of this paper is to detect best threshold level, advantage of the proposed technique is computed in a few times and segmented bands for the Gel Electrophoresis image correctly. On the other hand, the drawback of this technique removes the background, so some details may be lost.

Savelonas et al. (2012) proposed segmentation method for Gel Electrophoresis image as protein spots depend on active contours. The benefits of this method are solving some problems in image analysis such as noisy image; weak spots and the result have more quality.

Lee et al. (2011) proposed analysis of DNA Gel Electrophoresis image as spots using enhanced FCM for image segmentation. This paper compares basic FCM with Enhanced FCM. The benefits of this method bands are detected correctly depending on detecting lane that includes those bands and the repeated bands that have been removed. The weakness of this technique is because it detects bands, without segmenting it.

Raju and Rao (2013) established segmentation of the mammography image based on FCM and PSO techniques. PSO techniques had been used to improve the result of FCM. The advantages of FODPSO along with FCM are that they are the best techniques for medical image segmentation and as their computation time is very low.

Ghamisi et al. (2012) applied the DPSO technique to segment remote sensing image. The highest goal of this technique is to reach $(\mathrm{n}-1)$ an optimal result. The advantage of this technique is solving the problem of PSO that is trapped in local optima. It's also more efficiency compared with traditional PSO. This technique needs to be developed because it is the first time to be used in remote sensing image.

Sandeli and Batouche (2014) proposed new segmentation technique consists of PSO, GA and Artificial Bee Colony (ABC). This combination called generalized Island Model (GIM). The aim of this method is to solve the local optima problem. The consequence of this technique is beneficial, but when compared with DPSO and FODPSO, the latest technique is more accurate. This technique needs to be developed to enhance the system performance.

Ghamisi et al. (2014) established segmentation method depending on FODPSO along with Support Vector Machine (SVM) for remote sensing image to solve many optimization problems and Otsu problem. The advantages of this technique are reducing the n-level threshold to detect the optimal thresholds that maximize the variance between classes. Additionally, its computation time is very low. It is further more convenient than using DPSO for finding the global optimum. 


\section{Theoretical Background}

In this section, we will discuss several segmentation techniques that are applied to a gel electrophoresis image. These are Fuzzy Clustering Means (FCM) and swarm optimization techniques which produce accurate results in medical image segmentation. This paper discussed Particle Swarm Optimization (PSO), Darwinian Particle Swarm Optimization (DPSO) and Fractional Order Darwinian Particle Swarm Optimization (FODPSO) each one of those discovered to solve a problem in another.

\section{Fuzzy C-Means}

Cluster analysis is the primary method in pattern recognition, image processing and image segmentation. It depends on an unsupervised method. This technique is built on splitting data set $\mathrm{D}$ into small subset $\mathrm{d}$ (cluster). Clustering is the method of collecting similar objects into groups; it consists of two approaches: Hard clustering and fuzzy clustering (Yang, 1993). FCM one of the hard clustering (Dias et al., 2015). The hard clustering method depends on one cluster for each item in the data set while Fuzzy Clustering method is used if an item belongs to two or more clusters (Hemanth and Anitha, 2015). FCM stands for (Fuzzy C-Means), it is the principal method of clustering that depends on the fuzzy clustering theory. The first appearance of this method was in 1973 by Dunn and in 1981 this method was developed by Bezdek (Menon and Ramakrishnan, 2015; Yang and Huang, 2012). Minimizing the objective functions are the goal of this method. It also composes of iterations. The advantage of FCM is the results more accurate and one of the important disadvantages is executed in more time so you need to avoid this problem by using Particle Swarm Optimization and the extension of this method (Hemanth and Anitha, 2015). This technique works by dividing the image into two parts, one of its similar areas and different area. The goal of this method is to minimize the objective function (Alsmadi, 2015; Hemanth and Anitha, 2015). The objective function given by this equation:

$$
J=\sum_{i=1}^{c} \sum_{j=1}^{n} u_{i j}^{m} d_{i j}^{2}
$$

where, weight is $m \in[1, \infty], u_{i j}$ is between 0 and $1, c_{i}$ is the centroid of the cluster I, $d_{i j}, x_{j}$ is the distance between the center and data point given by this equation:

$$
d_{i j}=\left\|x_{j}-C_{i}\right\|
$$

To get an optimal objective function as in Equation 1 then we update membership by this equation:

$$
u_{i j}=\frac{1}{\sum_{k=1}^{c}\left[\frac{d_{i j}}{d_{k j}}\right]^{(2 / m-1)}}
$$

The FCM algorithm given by the following steps:

Step 1: Set value of $c, m$ randomly where $2 \leq c<m$.

Step 2: Put initial value for membership matrix $u^{k}=u^{0}$ using Equation 3 where is the number of iterations.

Step 3: Compute the cluster center using $C_{i}$ an equation:

$$
C_{i}=\frac{\sum_{j=1}^{n} u_{i j}^{m} x_{j}}{\sum_{j=1}^{n} u_{i j}^{m}}
$$

Step 4: Also by using Equation 3 update value for to $u^{k}$ compute $u^{k+1}$

Step 5: Compare between $u^{k+1}$ and $u^{k}$, if $\left\|u^{k+1}-u^{k}\right\|<\varepsilon$ then terminate; otherwise return to Step 3.

Note threshold value between 0 and 1 .

\section{SWARM}

In this subsection, we will discuss three segmentation methods depending on swarm intelligence: PSO, DPSO and FODPSO. Swarm Intelligence (SI) is helpful in several parts such as optimization. The main goal of the optimization method is to determine maximized or minimized objective functions in some feasible area (Talukder, 2011).

\section{Particle Swarm Optimization}

Particle Swarm Optimization (PSO) is a member of an effective and stochastic optimization algorithm. This technique is established by Eberhart and Kennedy in 1995 depending on the common behaviors of birds flocking and swarm theory (Rini et al., 2011). It is also useful for solving many optimization problems in several parts as image segmentation and its results are more accurate (Mohsen et al., 2011). The objective of PSO is to get the global optimal solution in a complex search space. Now, we found multiple version of PSO. These are assorted matches between this method and Genetic Algorithms (GA). The advantage of PSO is easier to compute and it is very faster than GA (Kaur and Singh, 2012). PSO is used in multiple fields such as signal processing, image segmentation, image processing, neural network, data mining and medical imaging (Tandan and Raja, 2013). It also consists of particles where particles set as candidate solutions. The aim of every particle is to produce an optimal solution in the search space. Traditional PSO algorithm consists of two main equations (Raju and Rao, 2013; Tandan and Raja, 2013): 


$$
\begin{aligned}
& v_{i+1}^{n}=m v_{i}^{n}+c_{1} r_{1}\left(\bar{g}_{i}^{n}-x_{i}^{n}\right)+c_{2} r_{2}\left(\bar{x}_{i}^{n}-x_{i}^{n}\right) \\
& +c_{3} r_{3}\left(\bar{n}_{i}^{n}-x_{i}^{n}\right) \\
& x_{i+1}^{n}=x_{i}^{n}+v_{i+1}^{n}
\end{aligned}
$$

At the beginning particle velocities are set to zero and set the particle at a random position.

Table 1 discusses factors that have been used in the Particle Swarm Optimization method. In "Equation 5" and "Equation 6" there are several parameters like velocity $v_{i}^{n}$, Particle position $x_{i}^{n}$, select random number $r_{1}, r_{2}, r_{3}$. Which the best value for all particles represented by $\bar{g}_{i}^{n}$, Local best is the best function for this particle and Neighborhood best is the best function for the neighborhood particle represented as the following $\bar{x}_{i}^{n}, \bar{n}_{i}^{n}$ also using constant values $m, c_{1}, c_{2}, c_{3}$.

The following algorithm explains how Particle Swarm Optimization method works.

Pseudocode of standard PSO considered as:

Yetirajam and Jena (2012; Cui et al., 2005; Raju and Rao, 2013)

Start particle with random position $x_{i}^{n}$ and velocity $v_{i}^{n}$

For all particles in search space from 1 to $n$

Do

Compute fitness value

Compare between fitness $\left(x_{i}\right)$ and fitness $\left(\bar{x}_{i}\right)$

If fitness $\left(x_{i}\right)>$ fitness $\left(\bar{x}_{i}\right)$

Then $\bar{x}_{i}=x_{i}$

Compare between fitness $\left(x_{i}\right)$ and fitness $\left(\bar{g}_{i}\right)$

If fitness $\left(x_{i}\right)>$ fitness $\left(\bar{g}_{i}\right)$

Then $\bar{g}_{i}=x_{i}$

Update velocity and position using Equation 5 and 6 End

The pervious pseudo code end when $\bar{g}_{i}$ is the optimal solution. The most common defects in PSO technique are dealing with problems PSO can solve one, but at the same time failed on another. Another defect PSO depends on the parameters, so any changes in one parameter can change at the speed of this technique.

Table 1. PSO Attributes in "Equation 5"and "Equation 6"

\begin{tabular}{ll}
\hline PSO attributes & Meaning \\
\hline$v_{i}^{n}$ & Particle velocity, ratio of particle chances \\
$r_{1}, r_{2}, r_{3}$ & Random number, usually between $(0,1)$ \\
$m, c_{1}, c_{2}, c_{3}$ & Constant values \\
$x_{i}^{n}$ & Particle position in search space \\
$\bar{x}_{i}^{n}$ & Local best \\
$\bar{g}_{i}^{n}$ & Global best, best value for all particles \\
$\bar{n}_{i}^{n}$ & Neighborhood best \\
\hline
\end{tabular}

\section{Darwinian Particle Swarm Optimization}

Optimization algorithms and PSO faced a common problem that may trap in a local optimum (Raju and Rao, 2013; Tillett and Rao, 2005). Darwinian Particle Swarm Optimization DPSO developed by Tillett and Rao (2005) to solve the problem of PSO that is escaping from local optima. Because of using one swarm of test solutions in PSO. It is complex for one swarm to differentiate between a local optimum and a global optimum, so DPSO was developed for solving this problem. For any time, many swarms are existing to test solutions. If a search gets to a local optimum, then the search in that area is substituted for another area in a search. DPSO is very beneficial compared with the PSO, DPSO produce result with efficient performance and in less CPU time than PSO.

PseudocodeofBasic DPSO technique (Ghamisi et al., 2012; Tillett and Rao, 2005)

For every swarm in the search space

Do

Apply the swarm (discussed below)

Move to new swarm

If the swarm is unsuccessful

Then remove it

End If

End

For every particle in the swarm

Do

Update Fitness Particle and Update Best Particle

If global best fitness is found

Then use a new particle

End If

If the swarm failed to reach best global fitness

Then a particle is removed

End If

End

\section{Fractional Order Darwinian Particle Swarm Optimization}

One extension of the Darwinian particle swarm optimization (DPSO) is FODPSO presented in Pires et al. (2010). This based on Fractional Calculus (Raju and Rao, 2013). Fractional Calculus (FC): Is the ideal topic for many researchers. The concept of fractional differential is taken from Grünwald Letnikov. This is given by the following equations (Couceiro et al., 2012; Kaur, 2012; Raju and Rao, 2013):

$D^{\alpha}[y(t)]=\lim _{i=0}\left[\frac{1}{i^{\alpha}} \sum_{m=0}^{+\infty} \frac{(-1)^{m} \Gamma(\alpha+1) y(t-m i)}{\Gamma(m+1) \Gamma(\alpha-m+1)}\right]$

where, $\alpha$ fractional coefficient $\alpha \in C, \Gamma$ is the gamma function and the $\mathrm{y}(\mathrm{t})$ represents a general signal. In Discrete time, the signal $D^{\alpha}[y(t)]$ can be defined as: 


$$
D^{\alpha}[y(t)]=\frac{1}{T^{\alpha}} \sum_{m=0}^{r} \frac{(-1)^{m}\lceil(\alpha+1) y(t-m T)}{\lceil(m+1)\lceil(\alpha-m+1)}
$$

The period for a sample is represented by $T$ and $r$ is the truncate order. The following equation depends on "Equation 1":

$$
\begin{aligned}
& D^{\alpha}\left[v_{i+1}^{n}\right]=c_{1} r_{1}\left(\bar{g}_{i}^{n}-x_{i}^{n}\right)+c_{2} r_{2}\left(\bar{x}_{i}^{n}-x_{i}^{n}\right) \\
& +c_{3} r_{3}\left(\bar{n}_{i}^{n}-x_{i}^{n}\right)
\end{aligned}
$$

Using the previous "Equation 9" and "Equation 5", "Equation 6" then "Equation 9" can be rewritten as the following:

$$
\begin{aligned}
& v_{i+1}^{n}=\alpha v_{i}^{n}+\frac{1}{2} \alpha v_{i-1}^{n}+\frac{1}{6} \alpha(1-\alpha) v_{i-2}^{n} \\
& +\frac{1}{24} \alpha(1-\alpha)(2-\alpha) v_{i-3}^{n}+c_{1} r_{1}\left(\bar{g}_{i}^{n}-x_{i}^{n}\right) \\
& +c_{2} r_{2}\left(\bar{x}_{i}^{n}-x_{i}^{n}\right)+c_{3} r_{3}\left(\bar{n}_{i}^{n}-x_{i}^{n}\right)
\end{aligned}
$$

DPSO can be seen as specific in case of the FO-DPSO when $\alpha=1$ in the previous equation (Kaur, 2012). Finally, FODPSO is faster than the PSO and more efficiently than DPSO in the area of avoiding local optima.

\section{Proposed Segmentation Technique}

This section contains 2 Subsections, each of which is the proposed steps applied in this study. First, discuss preprocessing steps to show how to convert the DNA sequence to gel electrophoresis image. Other Subsection explains segmentation steps that used for this type of image and state there in the pseudo code.

\section{Preprocessing Step}

In this subsection, we consider the steps to preprocessing DNA electrophoresis gel image. First, explore the algorithm to convert DNA sequences to gel images.

The procedure for creating gel electrophoresis image of DNA Sequence is sequenced by the following steps:

Step 1: Create a file, put multiple DNA sequences in this file and all of these must be in the same format like Fasta format.

Step 2: Apply DNA Sequence alignment ClustalW for each sequence in the file.

Step 3: Produced aligned file and apply a restriction enzyme to it that to cut DNA Sequence.

Step 4: Draw electrophoresis image: One gel for each sequence and one lane for each restriction enzyme.

In this subsection, we consider the steps to preprocessing DNA electrophoresis gel image. First, explore this algorithm applied to both multiple DNA sequences and the above steps to obtain the results for images which consist of lanes and bands. This algorithm can be applied by Bioperl toolbox and it is the first step for preprocessing image. Then we applied some MATLAB operations for image pre-processing such as: Image conversion from RGB color to grayscale if its color image, subtract background from an image, enhances it by applying some filter after this step, we apply some segmentation techniques such as: FCM, PSO, DPSO and FODPSO. Finally, we evaluate the result to be determined by the best technique.

\section{Segmentation Step}

Image segmentation is the main subject in image analysis, medical image processing and pattern recognition (Kannan et al., 2012; Mohsen et al., 2012; Yang and Huang, 2012). The target of image segmentation is dividing an image into several homogeneous regions as color, texture and detect boundary (Kannan et al., 2012; Wang and $\mathrm{Bu}, 2010$; Yang and Huang, 2012). There are several categories of image segmentation such as: Clustering based segmentation and edge based segmentation. This paper discusses FCM and PSO techniques for image segmentation. It is important to apply segmentation methods in the gel electrophoresis image to detect all bands and lanes and to use the resulted image to identify diseases such as breast cancer.

Pseudocode for the Proposed Segmentation Technique For each input image

Do

Read this image

If its color image

Then converts it to grayscale

End If

Enhance the result by applying specific filter to input image

Using techniques of segmentation to the enhanced image like segmentation threshold, FCM and swarm. Utilizing the results of three techniques and apply PSNR, MSE and others to evaluate it.

Compare the results from three techniques and obtain End the best result.

The preceding pseudo code describes proposed segmentation technique.

\section{Experimental Results}

In this section, the database consists of several images that have been segmented by proposing a segmentation technique in Fig. 3 using MATLAB tool. 
Results from the proposed segmentation technique are evaluated by image quality measurement factors and then the results are compared with each other to specify the accurate result. We will try four images that have been segmented to obtain the best result.

\section{Data Sets}

Data Sets are gathered from NCBI (National Center for Biotechnology Information). To retrieve data from NCBI uses http://www.ncbi.nlm.nih.gov/. We have tested 50 pictures of DNA sequence gel electrophoresis using MATLAB tool and Bioinformatics Toolbox. We have applied preprocessing method and specific segmentation techniques to obtain our results. Each image consists of multiple lanes which are vertical and there are several horizontal bands for every lane. All of these images are tested by specific segmentation methods are discussed in this section. Our data set presented as images with different types like PNG and JPG and different sizes because images are real. Most of them grayscale image. In this study used 4 images from data sets to be presented. The 4 images are grayscale named as '15.png', '22.png', '7.jpg' and '35.png'. The dimension of them respectively $(673 * 186),(489 * 190)$, $(553 * 931)$ and $(599 * 643)$.

\section{Performance Evaluation}

Pseudocode of the proposed segmentation techniques has been implemented using the MATLAB R2012a tool. Multiple electrophoresis images have been used in our experiments can be tested. The following figure shows various segmentation methods are applied to the image ' 15 ' which type is PNG image.

The previous figure shows various image segmentation methods all of which are applied on the specific image '15.png'. After preprocessing and enhancement of this image using a specific filter, the output image is tested by various segmentation techniques such as an applied segmentation threshold; FCM uses threshold, FCM, PSO, DPSO and FODPSO. Results from PSO $4 \mathrm{f}$ and DPSO $4 \mathrm{~g}$ produce the same result which is very good for all methods, but the best result of all techniques used in this image is FODPSO 4h. In this Fig. 4h which produces very high PSNR and all bands in the image that are segmented.

The following figure tested all of segmentation methods on image '22.png'.

In this figure result produced from FODPSO $5 \mathrm{~h}$ is the best.

The following figure tested all of segmentation methods on image '7.jpg'.

In this figure, the result produced from FODPSO 6 $\mathrm{h}$ is the best.

We apply segmentation methods on '35.png'.

In the previous figure FODPSO $7 \mathrm{~h}$ produce very good image.

\section{Comparison with Other Techniques}

In this subsection, we evaluated the quality of previously discussed figures in the tables that represent MSE, PSNR and other factors. We have noticed that when MSE is low, PSNR is very high, so, there is a very low error in the image.

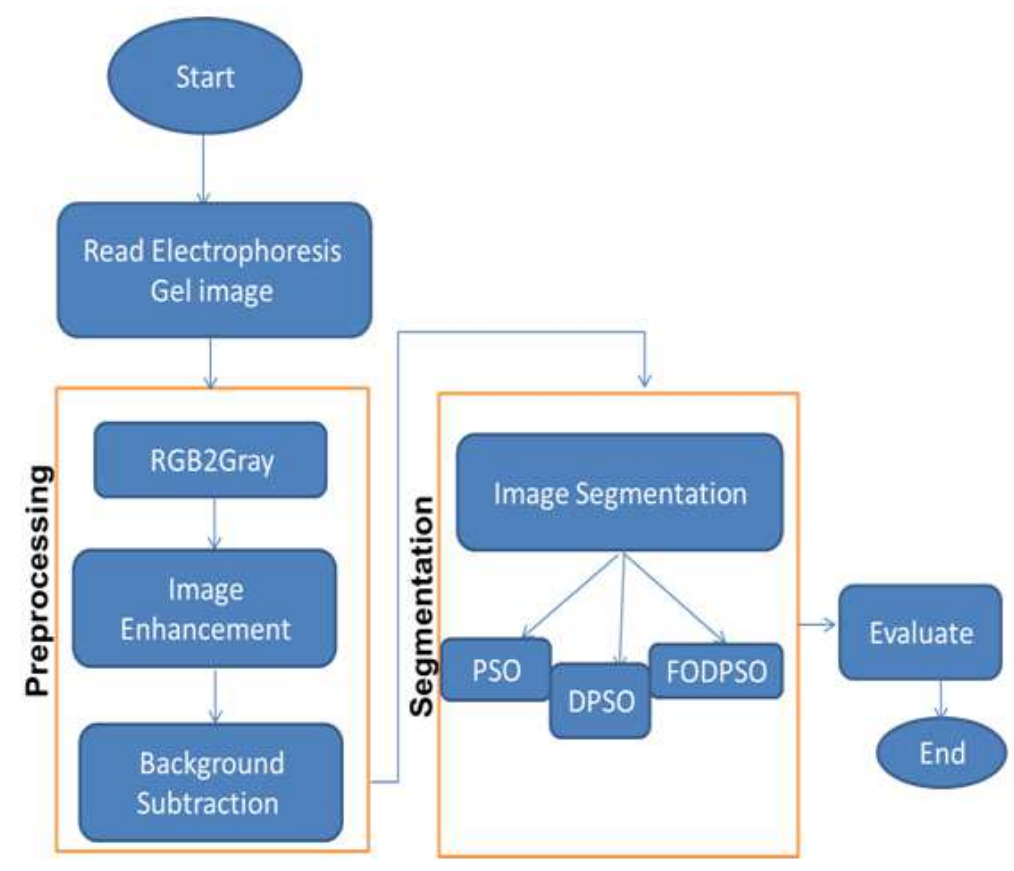

Fig. 3. Block diagram of proposed segmentation technique 


\section{Mean Squared Error (MSE)}

The squared difference between the original image and segment image. MSE measured error between pre-processing reference image and segmented image, the large value means poor quality image (Desai and Kulkarni, 2010; Ece and Mmu, 2011). It is defined by:

$M S E=\frac{1}{M N} \sum_{i=1}^{M} \sum_{k=1}^{N}(x(i, k)-\bar{x}(i, k))^{2}$

where, $x(i, k)$ is represent preprocessing reference image and $\bar{x}(i, k)$ is the segmented image.

\section{Peak Signal to Noise Ratio (PSNR)}

Used to measure quality between two images after applying some operation on them such as image compression, image enhancement and image segmentation (Desai and Kulkarni, 2010; Ece and Mmu, 2011). It's computed by calculating MSE first and if the value of PSNR is small, then this means that the image has poor quality:

$$
P S N R=10 \log _{10}[255 / M S E]
$$

\section{Average Difference (AD)}

Computes the difference between the original image and segment image and then take the average result. It is based by this equation (Desai and Kulkarni, 2010; Ece and Mmu, 2011):

$$
A D=\frac{1}{M N} \sum_{i=1}^{M} \sum_{k=1}^{N}(x(i, k)-\bar{x}(i, k))
$$

\section{Maximum Difference (MD)}

Computes differences between the original image and segment image and then take the maximum value of it (Desai and Kulkarni, 2010; Ece and Mmu, 2011), if the value of MD is large this mean that the image has poor quality:

$$
M D=\max |x(i, k)-\bar{x}(i, k)|
$$

\section{Normalized Absolute Error (NAE)}

Is defined by following equation (Desai and Kulkarni, 2010; Ece and Mmu, 2011):

$$
N A E=\frac{\sum_{i=1}^{M} \sum_{k=1}^{N}|x(i, k)-\bar{x}(i, k)|}{\sum_{i=1}^{M} \sum_{k=1}^{N}|x(i, k)|}
$$

\section{Structural Content (SC)}

Measures the similarity between two images, is considered one type of correlation (Desai and Kulkarni, 2010; Ece and Mmu, 2011):

$$
S C=\frac{\sum_{i=1}^{M} \sum_{k=1}^{N} x(i, k)^{2}}{\sum_{i=1}^{M} \sum_{k=1}^{N} \bar{x}(i, k)^{2}}
$$

\section{Normalized Cross-Correlation (NK)}

It is also a type of correlation. It computes the similarity between the original image and segment image (Ece and Mmu, 2011).

$$
N K=\frac{\sum_{i=1}^{M} \sum_{k=1}^{N} x(i, k) \bar{x}(i, k)}{\sum_{i=1}^{M} \sum_{k=1}^{N} x(i, k)^{2}}
$$

Using Equation 11, 12, 13, 14, 15, 16 and 17 for all of the above in Fig. 4.

In Fig. 8 comparison of image quality measures of the various image segmentation method applied to image '15.png'. Represented resulted images after applying proposed segmentation technique represented in and the evaluation of each resulted image by parameters of image quality measurements. The horizontal bar show resulted images using segmentation techniques as segmentation using threshold, FCM uses threshold, FCM, PSO, DPSO and FODPSO for the same image such as image '15.png'.

The vertical bar presents the evaluation parameters such as MSE, PSNR, NK, AD, SC, MD and NAE discussed above. It can be seen that the value of Mean Squared Error (MSE) decreased for all images which is so good for the result, so in image '15FO-DPSO' is the best result for all using MSE. Using Peak Signal to Noise Ratio (PSNR) seen that the values for their increased for all images, from the definition of PSNR it is so good. Also, the best result by using PSNR as parameter is image ' $15 \mathrm{FO}-\mathrm{DPSO}$ '.As the same for all parameters image resulted from FODPSO is the best result.

In Fig. 9 comparison of image quality measures of the various image segmentation method applied to image '22.png'. This bar chart illustrates resulted images after applying proposed segmentation technique represented in and the evaluation of each resulted image by parameters of image quality measurements. The horizontal bar show resulted 
images using segmentation techniques as segmentation using threshold, FCM uses threshold, FCM, PSO, DPSO and FODPSO for the same image such as image '22.png'.

The vertical bar presents the evaluation parameters such as MSE, PSNR, NK, AD, SC, MD and NAE discussed above. It can be seen that the FCM produced good results, but the resulted images from PSO, DPSO, FODPSO produced the same result which is high quality and the best comparing with other images. Also parameters such as MSE, PSNR, NAE, AD, NK and MD determined that the PSO, DPSO, FODPSO produce the best results for the image '22.png'.

In Fig. 10 comparison of image quality measures of the various image segmentation method applied to image '7.jpg'. This bar chart illustrates resulted images after applying proposed segmentation technique represented in and the evaluation of each resulted image by parameters of image quality measurements. The horizontal bar show resulted images using segmentation techniques as segmentation using threshold, FCM uses threshold, FCM, PSO, DPSO and FODPSO for the same image such as image '7.jpg'.

The vertical bar presents the evaluation parameters such as MSE, PSNR, NK, AD, SC, MD and NAE discussed above. It can be seen that the FCM produced good results, but the resulted images from FODPSO produced the best result which is the lowest MSE value, highest PSNR value, smallest NK and the smallest NAE.

In Fig. 11 comparison of image quality measures of the various image segmentation method applied to image '35.png'. This bar chart illustrates resulted images after applying proposed segmentation technique represented in and the evaluation of each resulted image by parameters of image quality measurements. The horizontal bar show resulted images using segmentation techniques as segmentation using threshold, FCM uses threshold, FCM, PSO, DPSO and FODPSO for the same image such as image '35.png'.

The vertical bar presents the evaluation parameters such as MSE, PSNR, NK, AD, SC, MD and NAE discussed above. It can be seen that the FCM produced good results, but the resulted images from DPSO, FODPSO produced the best result, which present the same result. Are the lowest MSE value, highest PSNR value, smallest NK and the smallest NAE. All of the above figures show that FODPSO produced more accurate result that segment all lanes and bands. This produces highest PSNR value and lower MSE compared with other technique discussed in this study.

\section{Discussion}

Segmentation methods such as threshold, FCM and FCM uses threshold have shown some weakness in segmenting all bands and lanes in images, so some bands not clear using the previous methods. To overcome such disadvantages, swarm optimization techniques like PSO, DPSO and FODPSO have applied for the same images which provide optimal, effective and high accuracy result compared with the pervious techniques. For experiment several images have tested and select four images labeled '15.png', '22.png', '7.jpg' and '35.png' put all of those images in figure using the segmentation methods explained before labeled Fig. 4 to 7 respectively, for the four images. Using factors for measuring error in result images representing in tables labeled from the Fig. 8 to 11 of the four tested image. Each table consists of six images after applying segmentation methods and the value of factors for each technique result.

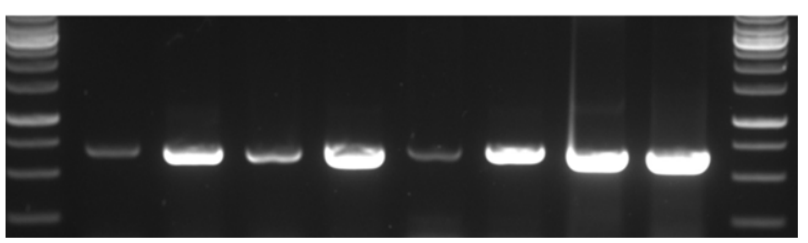

(a)

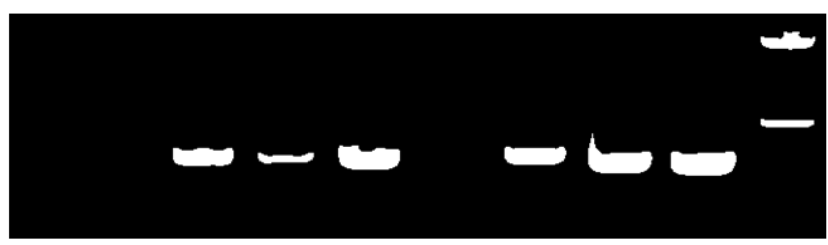

(c)

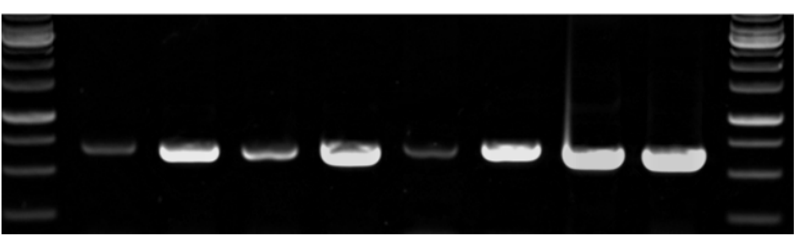

(b)

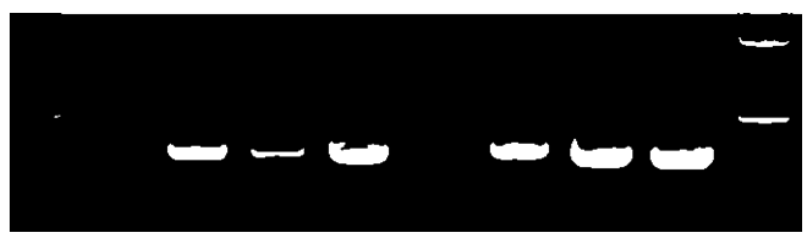

(d) 


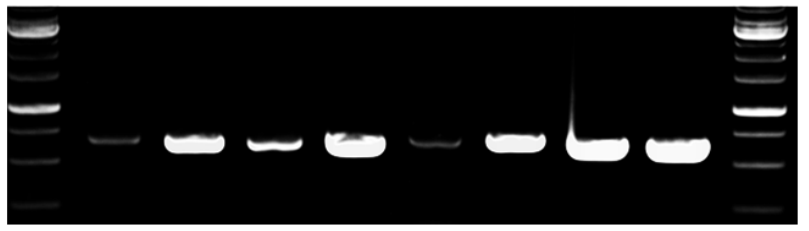

(e)

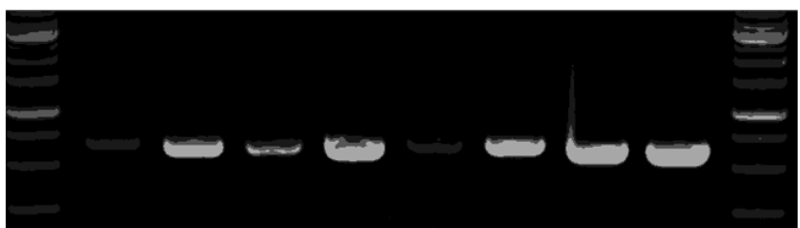

(g)

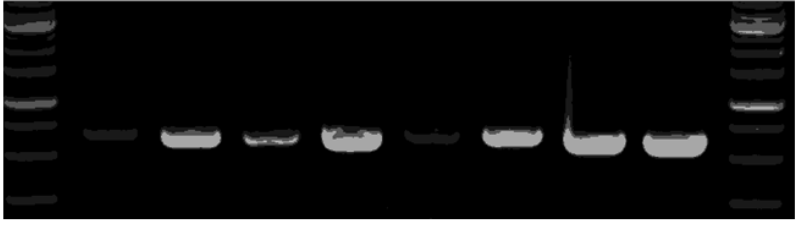

(f)

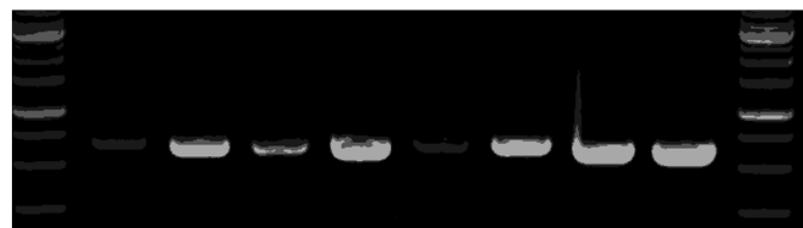

(h)

Fig. 4. Apply segmentation technique on image 15; (a) Original image; (b) After pre-processing; (c) Using threshold segmentation; (d) Using FCM thresholding; (e) Using FCM; (f) Using PSO; (g) Using DPSO; (h) Using FODPSO

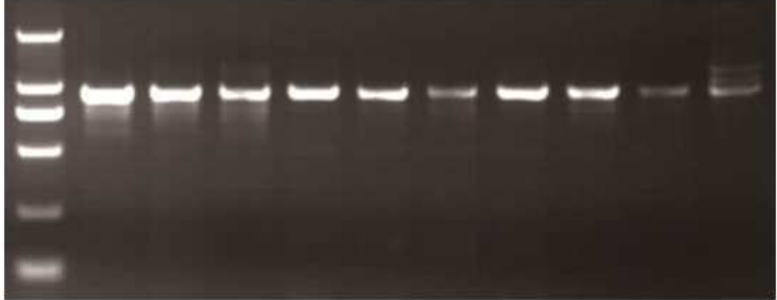

(a)

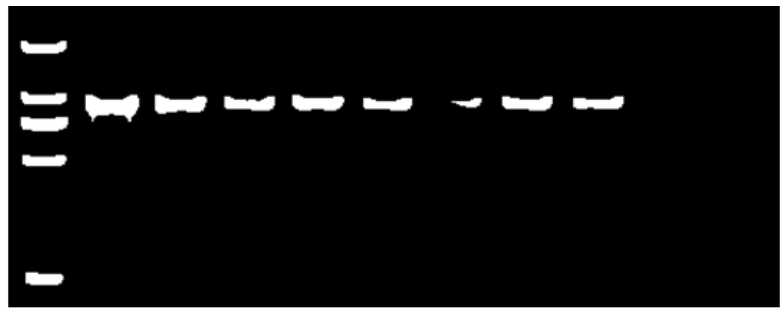

(c)

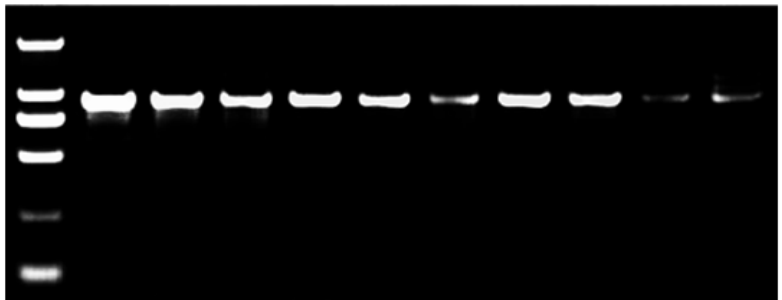

(e)

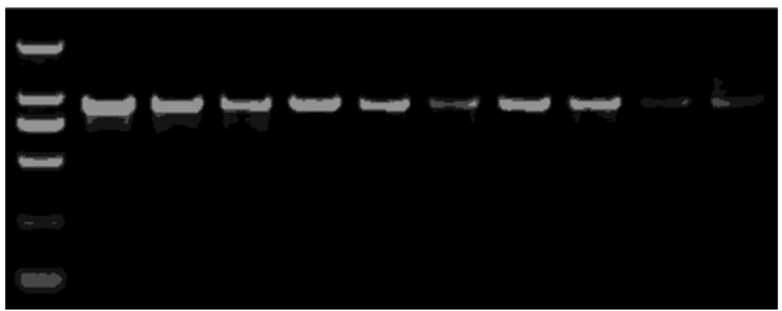

(g)

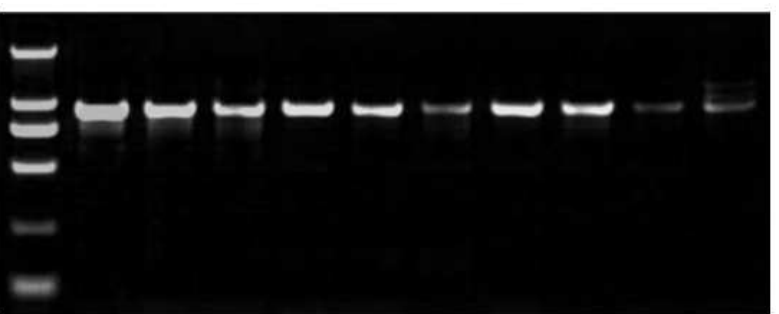

(b)

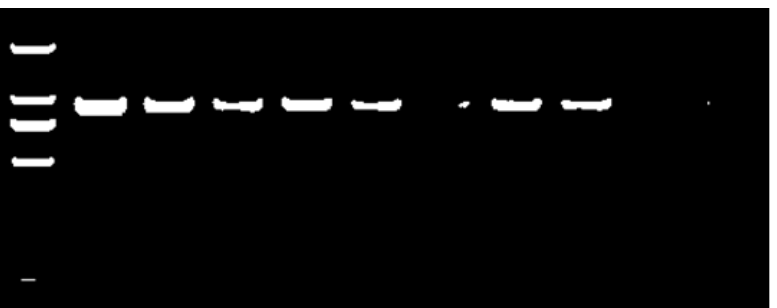

(d)

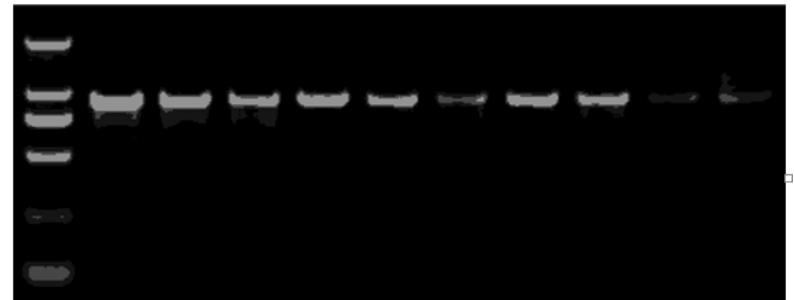

(f)

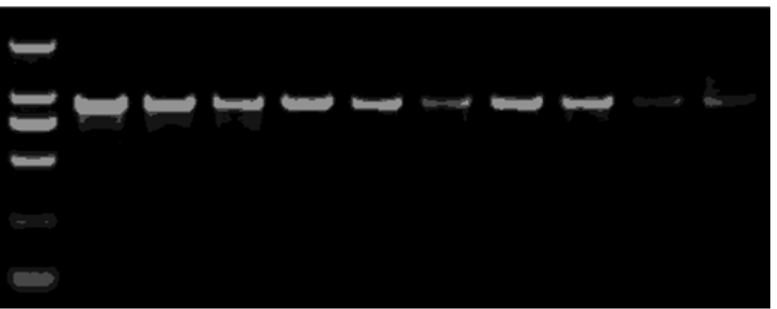

(h)

Fig. 5. Apply segmentation technique on image 22; (a) Original image; (b) after preprocessing; (c) Using threshold segmentation; (d) Using FCM thresholding; (e) Using FCM; (f) Using PSO; (g) Using DPSO; (h) Using FODPSO 


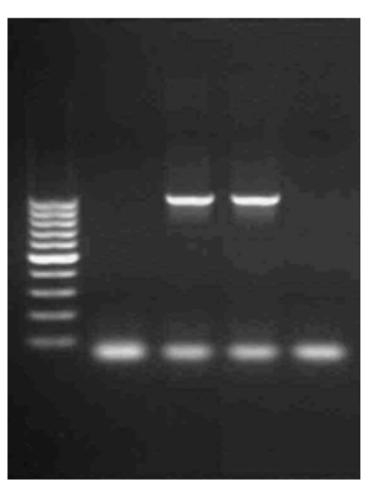

(a)

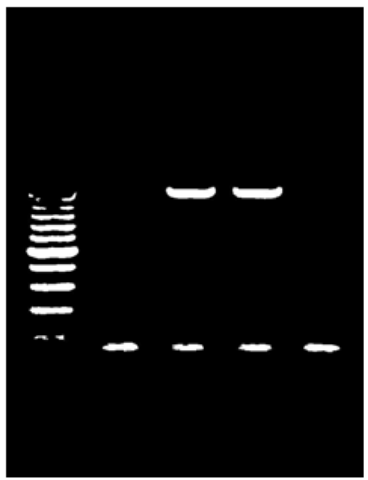

(d)

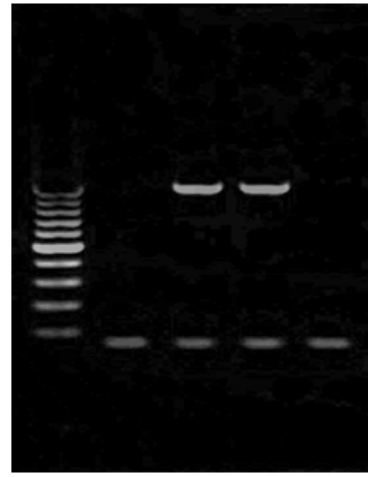

(b)

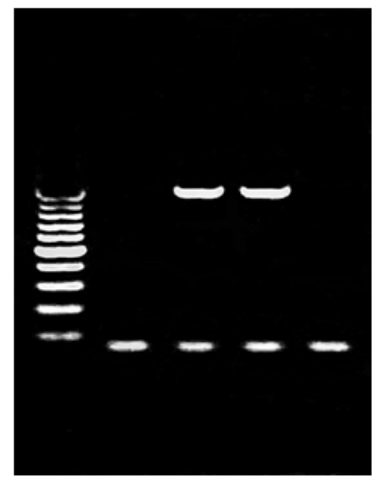

(e)

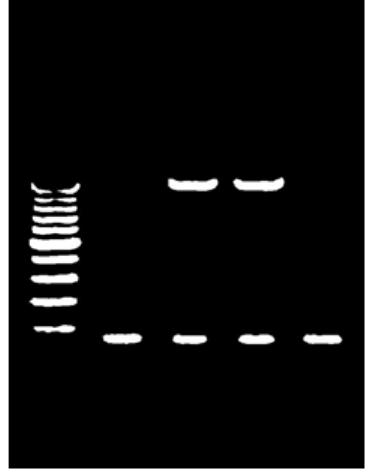

(c)

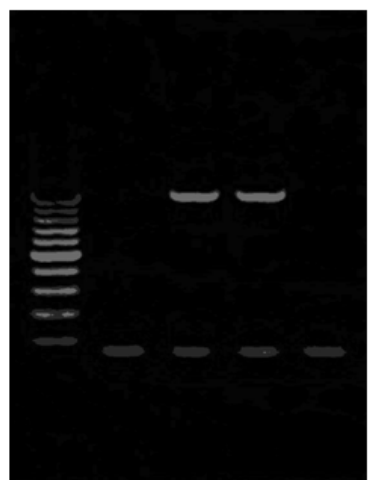

(f)

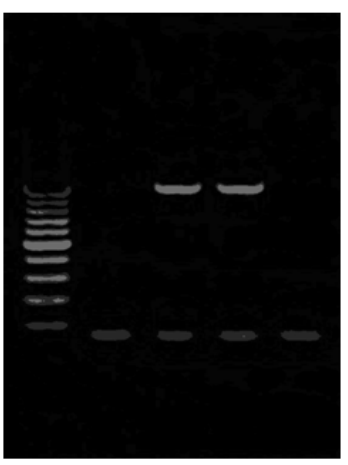

(g)

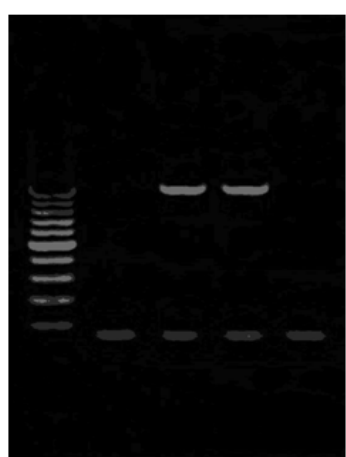

(h)

Fig. 6. Apply segmentation technique on image 7; (a) Original image; (b) After Pre-processing; (c) Using threshold segmentation; (d) Using FCM thresholding; (e) Using FCM (f) Using PSO; (g) Using DPSO; (h) Using FODPSO

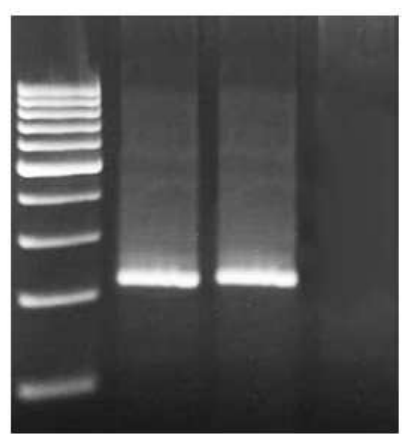

(a)

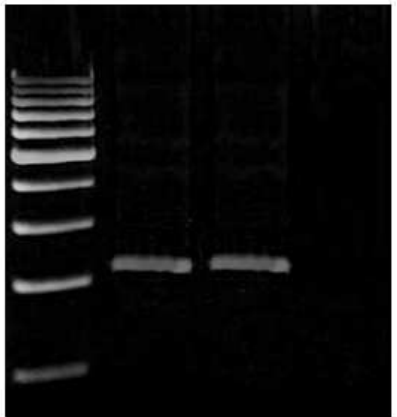

(b)

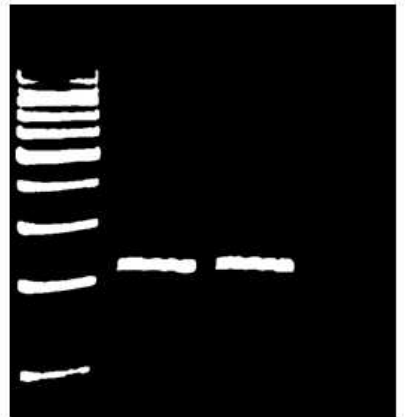

(c) 


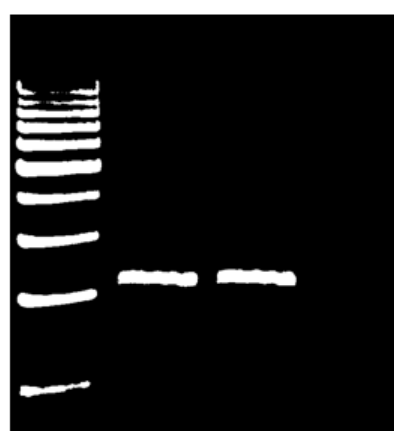

(d)

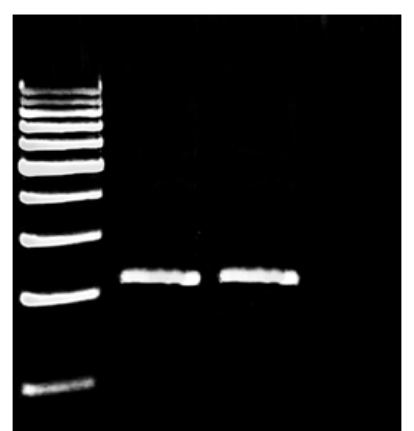

(e)

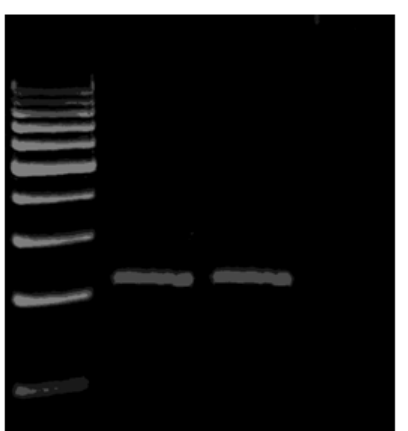

(f)

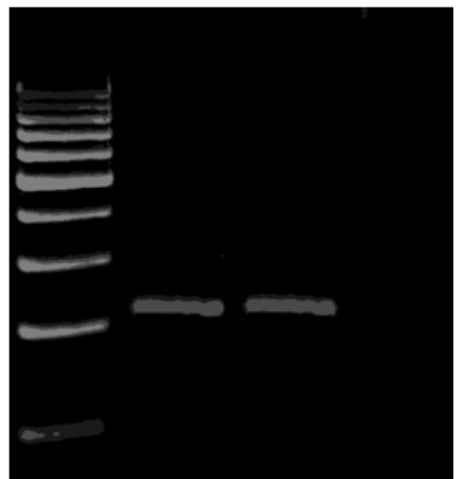

(g)

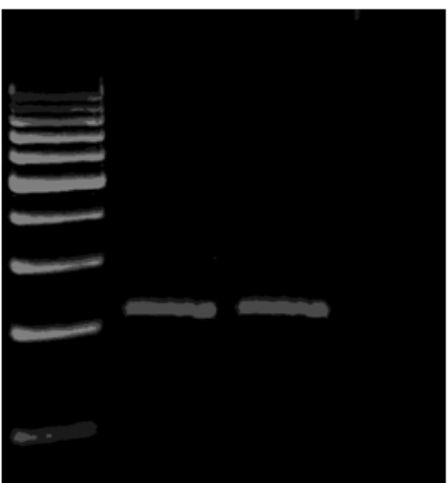

(h)

Fig. 7. Apply segmentation technique on image 35; (a) Original image; (b) Afterpre-processing; (c) Using threshold segmentation; (d) Using FCM thresholding; (e) Using FCM; (f) Using PSO; (g) Using DPSO; (h) Using FODPSO

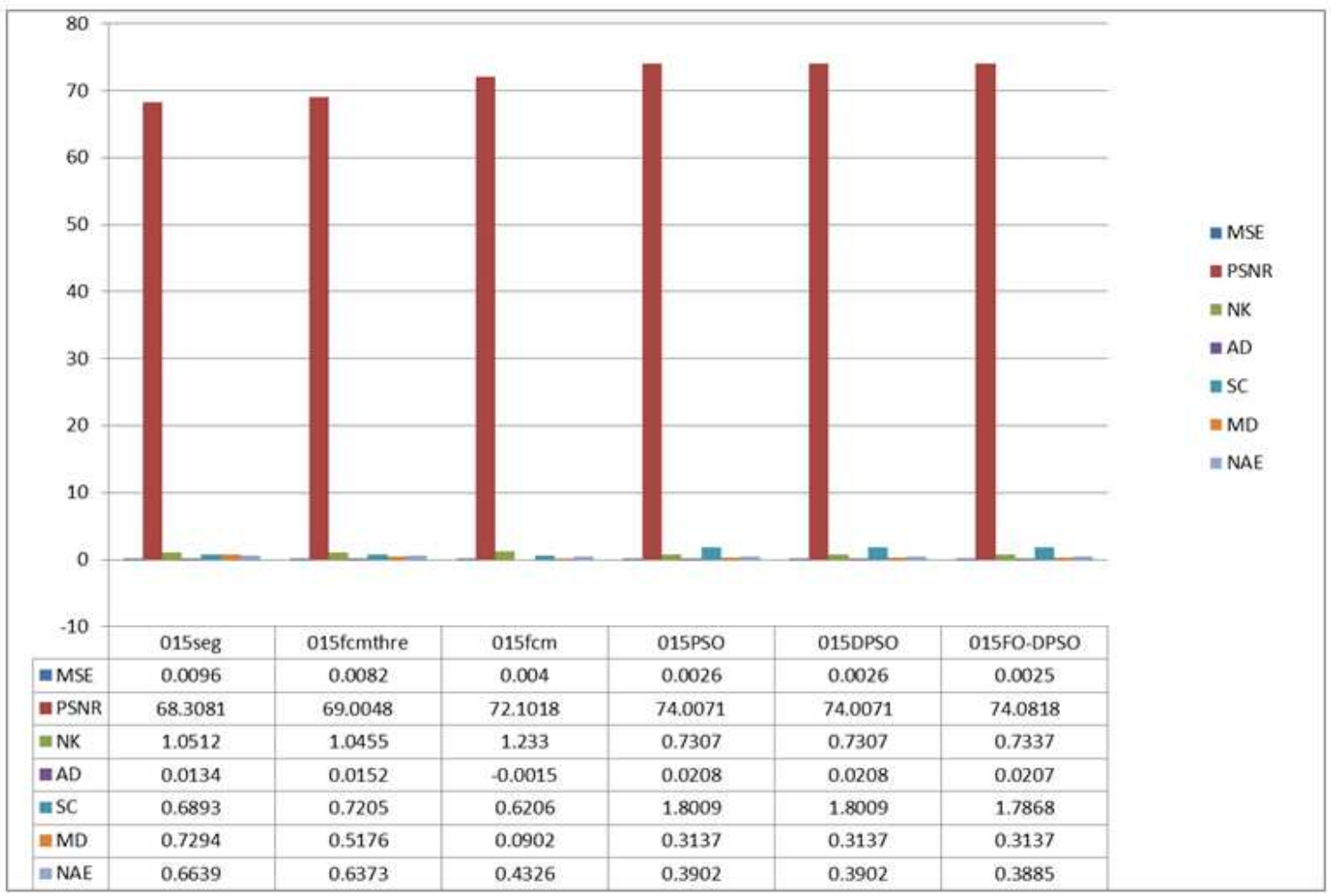

Fig. 8. Comparison of image quality measures of the various image segmentation method applied to image '15.png' 


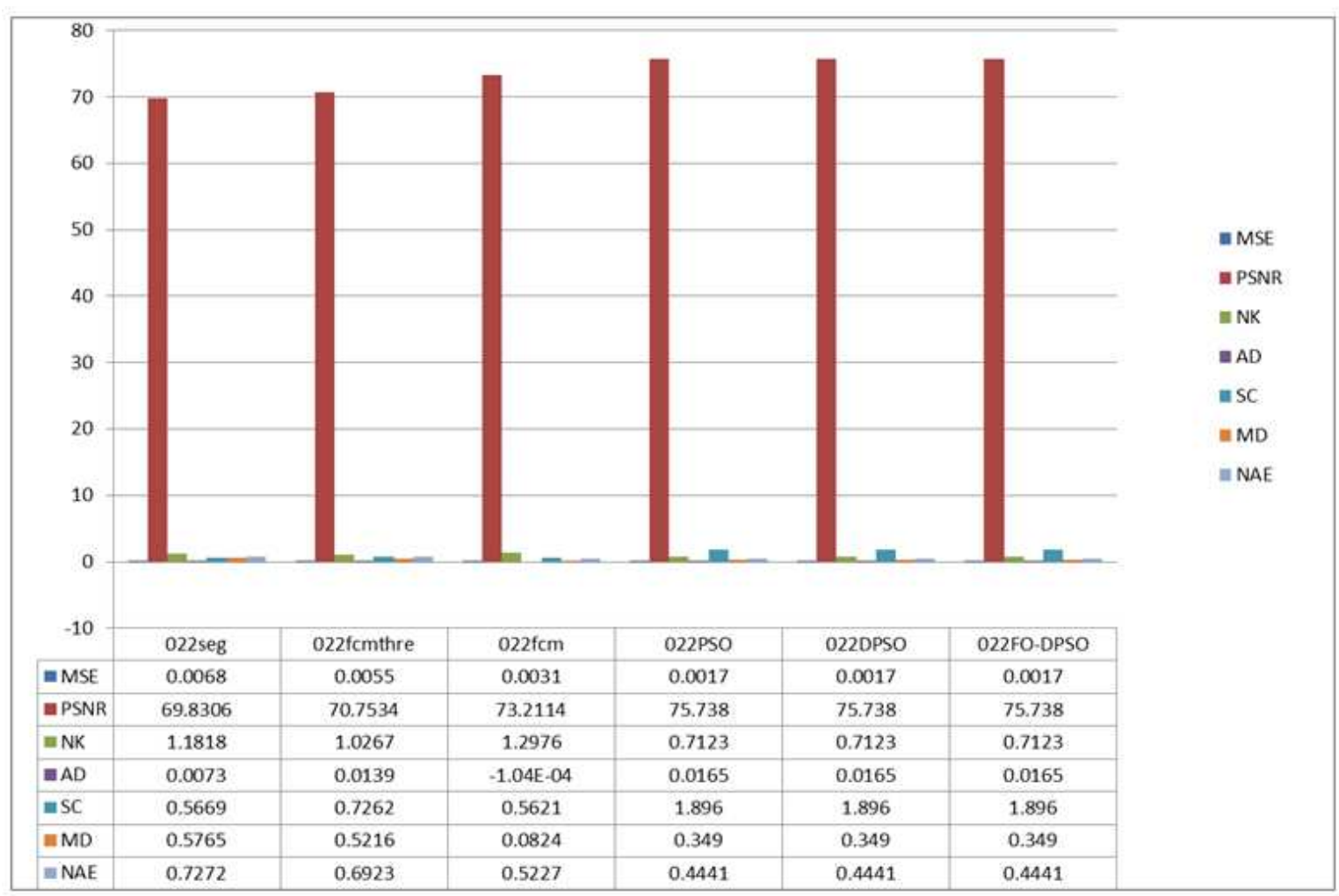

Fig. 9. Comparison of image quality measures of the various image segmentation method applied to image '22.png'

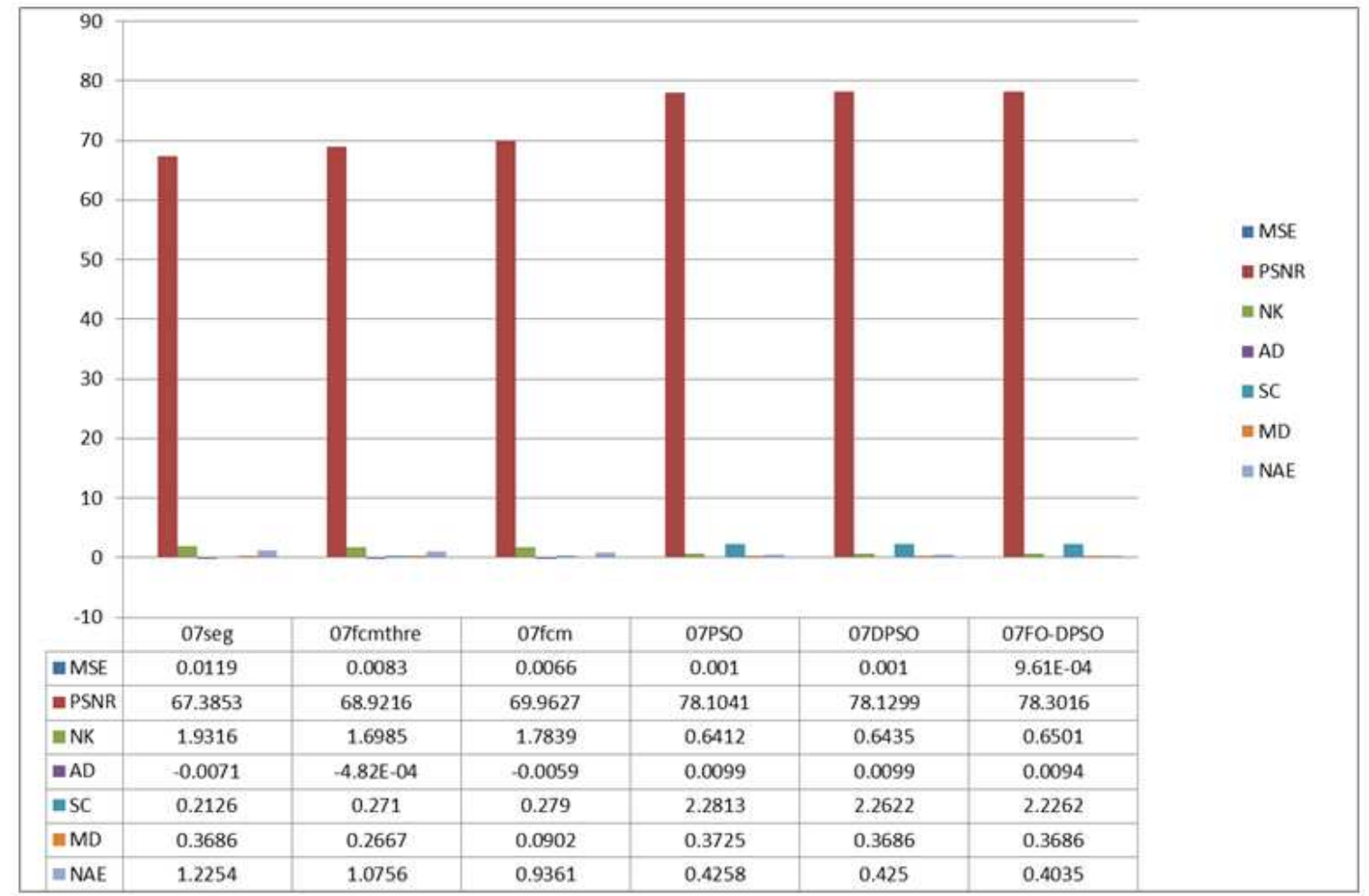

Fig. 10. Comparison of image quality measures of the various image segmentation method applied to image '7.jpg' 


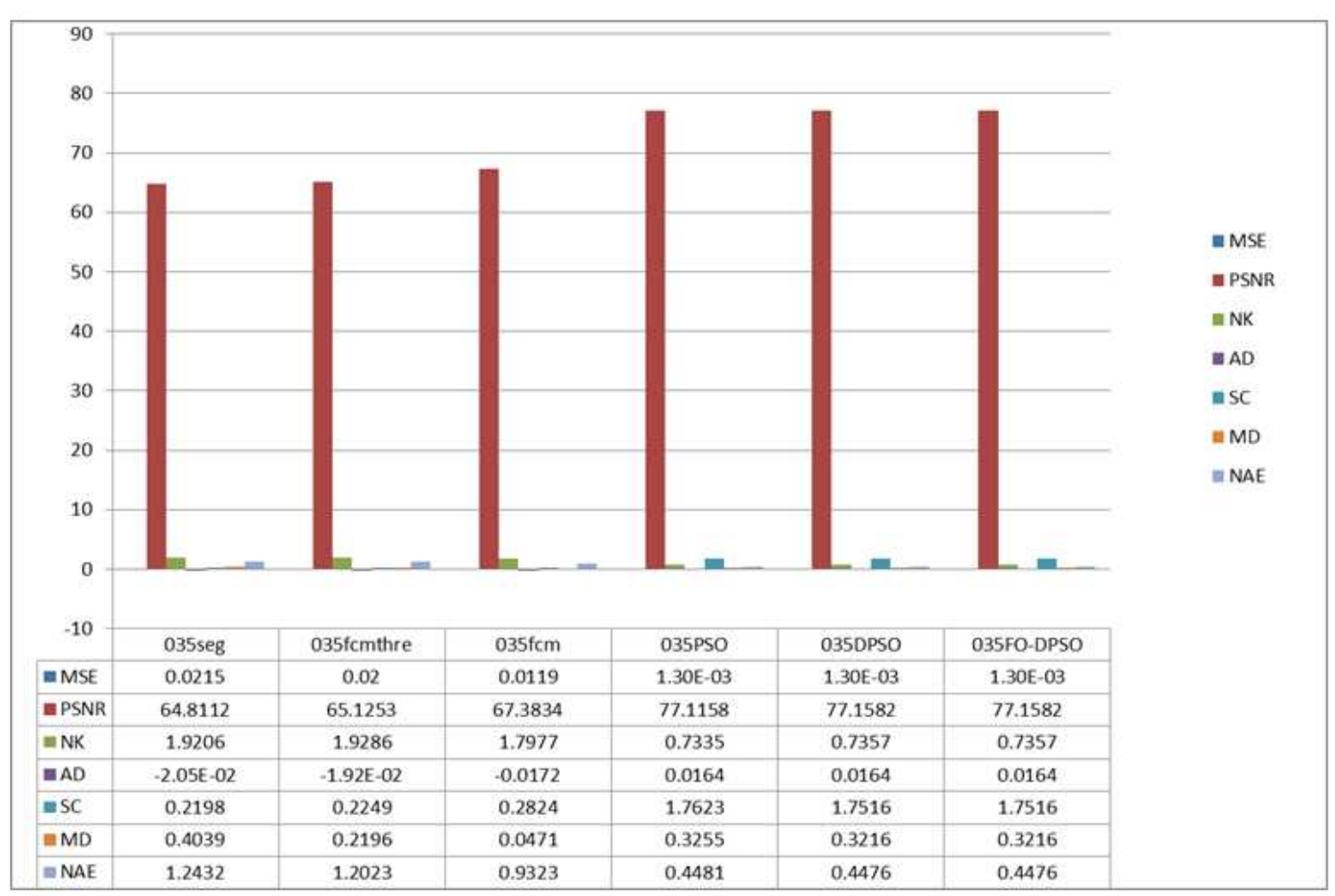

Fig. 11. Comparison of image quality measures of the various image segmentation method applied to image '35.png'

For example, using image '15.png' after applying preprocessing to it apply threshold segmentation which produces image named 'seg15.png' this result is poor it's PSNR $=68.3081 \mathrm{~dB}$. Apply FCM threshold of the preprocessing image which produce image named '15FCMthre.png' the result is very poor it's PSNR = $69.0048 \mathrm{~dB}$. Apply FCM to the pre-processing image which produce image named '15FCM.png' the result is good it's PSNR $=72.1018 \mathrm{~dB}$. After applying the proposed approach using swarm produce high accuracy results such as '15PSO.png' and '15DPSO.png' the results of PSO and DPSO respectively, are very good comparing with the previous methods which produce the same results PSNR $=74.0071 \mathrm{~dB}$. The best result with a high accuracy image named '15FODPSO.png' which applied FODPSO it's PSNR $=74.0818 \mathrm{~dB}$ representing in Fig. $4 \mathrm{~h}$.

For image '22.png' after applying the same techniques in Fig. 5 and 9 comparisons between results for all pervious segmentation methods and the proposed methods. The result from PSO, DPSO and FODPSO represented as '22PSO.png', '22DPSO.png' and '22FODPSO.png' produce the high accuracy and more effective result and produce the same PSNR. PSNR = $75.738 \mathrm{~dB}$, represented in Fig. $5 \mathrm{f}$ to $5 \mathrm{~h}$.

Using the same techniques on images '7.jpg' and '35.png' in Fig. 6 and 7 respectively. In Fig. 6 and 10 the result image from using FODPSO technique named as '7FODPSO.jpg' represented in Fig. 6h produces the best result, it's PSNR $=78.3016 \mathrm{~dB}$.
In Fig. 7 and 11 images named '35DPSO.png' and '35FODPSO.png' represented in Fig. $7 \mathrm{~g}$ and $7 \mathrm{~h}$ produce the best result and efficient compared with other techniques and produce the same PSNR. PSNR $=77.1582 \mathrm{~dB}$.

\section{Conclusion}

Image segmentation methods for segmenting and detecting all bands of Gel electrophoresis images representing the DNA sequence is proposed and implemented. This work focuses on using several techniques for image segmentation and compare between them to find the best technique which produce the best results. The method is based on using threshold segmentation, FCM, FCM uses a threshold to segment Gel Electrophoresis images note the results and then compare their results after applying the proposed technique using swarm optimization and their generations like PSO, DPSO and FODPSO. PSO and DPSO techniques are quite effective. The benefits of FODPSO are decreasing the computational time and more efficient than DPSO in area of avoiding local optima. The experiments demonstrate that the image segmented using the proposed technique by FODPSO represent high accuracy, effective result and best technique to segment and detect all bands. In the future, We can extend this technique in DNA image classification to diagnose diseases. 


\section{Funding Information}

The authors have no support or funding to report.

\section{Author's Contributions}

Sara Ibrahim Ibrahim: Participated in sample collection and performed data analyses. Drafted the manuscript, designed the experiment, write the MS, thoroughly revised and modified the MS.

Mohamed Abd Allah Makhlouf: Participated in sample collection and read and approved the manuscript.

Ghada.S. El-tawel: Designed the research plan and organized the study, read and approved the manuscript.

M.E. Wahed: Conceived this study, read and approved the manuscript.

\section{Ethics}

This article is original and contains unpublished material. The corresponding author confirms that all of the other authors have read and approved the manuscript and no ethical issues involved.

\section{References}

Ahmad, A.R., Z. Hussain, F. Ahmad, M.H.M. Noor and S.Z. Yahaya, 2013. Gel electrophoresis image segmentation with kapur method based on particle swarm optimization. Proceedings of the 5th International Conference on Computational Intelligence, Communication Systems and Networks, Jun. 5-7, IEEE Xplore Press, Madrid, pp: 393-396. DOI: $10.1109 /$ CICSYN.2013.60

Akhter, N., A. Khan, Y. Talib, S. Shadab and R. Patel, 2008. Analysis of gel electrophoresis images. Proceedings of the 1st International Conference on Emerging Trends in Engineering and Technology, Jul. 16-18, IEEE Xplroe Press, Nagpur, Maharashtra, pp: 106-109.

DOI: 10.1109/ICETET.2008.132

Alsmadi, M.K., 2015. MRI brain segmentation using a hybrid artificial bee colony algorithm with fuzzyc mean algorithm. J. Applied Sci., 15: 100-109. DOI: $10.3923 /$ jas.2015.100.109

Couceiro, M.S., F.M.L. Martins, R.P. Rocha and N.M.F. Ferreira, 2012. Introducing the fractional order robotic Darwinian PSO. Proceedings of the AIP Conference, Jul. 10-14, AIP, Vienna, Austria, pp: 242-251. DOI: 10.1063/1.4765496

Cui, X., T.E. Potok and P. Palathingal, 2005. Document clustering using particle swarm optimization. Proceedings of the IEEE Swarm Intelligence Symposium, Jun. 8-10, IEEE Xplore Press, Pasadena, CA, USA., pp: 185-191.

DOI: $10.1109 /$ SIS.2005.1501621
Desai, S.D. and L. Kulkarni, 2010. A quantitative comparative study of analytical and iterative reconstruction techniques. Int. J. Image Process., 4: 307-319.

Dias, M., G. Maydana and M.D. Aguiar, 2015. An extension of fuzzy c-means applied to spot recognition in images of two-dimensional electrophoresis. Proceedings of the 14th Mexican International Conference on Artificial Intelligence, IEEE Xplore Press, Cuernavaca, pp: 58-64. DOI: 10.1109/MICAI.2015.16

Ece, C. and M. Mmu, 2011. Image quality assessment techniques PN spatial domain. IJCST, 2: 177-184.

França, L.T., E. Carrilho and T.B. Kist, 2002. A review of DNA sequencing techniques. Q. Rev. Biophys., 35: 169-200. DOI: 10.1017/S0033583502003797

Ghamisi, P., M.S. Couceiro, N.M.F. Ferreira and L. Kumar, 2012. Use of Darwinian particle swarm optimization technique for the segmentation of Remote Sensing images. Proceedings of the IEEE International Geoscience and Remote Sensing Symposium, Jul. 22-27, IEEE Xplore Press, Munich, pp: 4295-4298. DOI: 10.1109/IGARSS.2012.6351718

Ghamisi, P., S. Member, M.S. Couceiro, S. Member and F.M.L. Martins et al., 2014. Multilevel image segmentation based on fractional-order Darwinian particle swarm optimization. IEEE Trans. Geosci. Remote Sens., 52: 2382-2394. DOI: $10.1109 /$ TGRS.2013.2260552

Hemanth, D.J. and J. Anitha, 2015. Performance improved modified fuzzy c-means algorithm for abnormal magnetic resonance brain image segmentation. Informatica, 26: 635-648.

Kannan, S., S. Ramathilagam, R. Devi and E. Hines, 2012. Strong fuzzy c-means in medical image data analysis. J. Syst. Software, 85: 2425-2438. DOI: $10.1016 /$ j.jss.2011.12.020

Kaur, A. and M. Singh, 2012. An overview of PSObased approaches in image segmentation. Int. J. Eng. Technol., 2: 1349-1357.

Kaur, J., 2012. Integration of clustering, optimization and partial differential equation method for improved image segmentation. Int. J. Image Graph. Signal Process., 4: 26-33.

Lee, J.D., C.H. Huang, N.W. Wang and C.S. Lu, 2011. Automatic DNA sequencing for electrophoresis gels using image processing algorithms. J. Biomed. Sci. Eng., 4: 523-528. DOI: 10.4236/jbise.2011.48067

Menon, N. and R. Ramakrishnan, 2015. Brain Tumor Segmentation in MRI images using unsupervised Artificial Bee Colony algorithm and FCM clustering. Proceedings of the International Conference on Communications and Signal Processing, Apr. 2-4, IEEE Xplore Press, Melmaruvathur, pp: 6-9.

DOI: 10.1109/ICCSP.2015.7322635 
Mohsen, F., M.M. Hadhoud, K. Moustafa and K. Ameen, 2012. A new image segmentation method based on particle swarm optimization. Int. Arab J. Inf. Technol., 9: 487-493.

Mohsen, F.M., M.M. Hadhoud and K. Amin, 2011. A new optimization-based image segmentation method by particle swarm optimization. Int. J. Adv. Comput. Sci. Applic.

Nelson, R., S. Foo and M. Weatherspoon, 2008. Using hidden Markov modeling in DNA sequencing. Proceedings of the 40th Southeastern Symposium on System Theory, Mar. 16-18, IEEE Xplore Press, New Orleans, LA., pp: 215-217. DOI: $10.1109 /$ SSST.2008.4480223

Noor, M.H.M., Z. Hussain, K.A. Ahmad and A.R. Ainihayati, 2011. Gel electrophoresis image segmentation with Otsu method based on particle swarm optimization. Proceedings of the IEEE 7th International Colloquium on Signal Processing and its Applications, Mar. 4-6, IEEE Xplore Press, Penang, pp: 426-429. DOI: $10.1109 /$ CSPA.2011.5759915

Pires, E.J., J.A. Machado, P.B. Cunha and L. Mendes, 2010. Particle swarm optimization with fractionalorder velocity. J. Nonlinear Dynam., 61: 295-301.

Raju, N.G. and P.A.N. Rao, 2013. Particle swarm optimization methods for image segmentation applied in mammography. J. Eng. Res. Applic., 3: 1572-1579.

Rini, D.P., S.M. Shamsuddin and S.S. Yuhaniz, 2011. Particle swarm optimization: Technique, system and challenges. Int. J. Comput. Applic., 14: 19-26. DOI: $10.5120 / 1810-2331$

Sandeli, M. and M. Batouche, 2014. Multilevel thresholding for image segmentation based on parallel distributed optimization. Proceedings of the 6th International Conference of Soft Computing and Pattern Recognition, Aug. 11-14, IEEE Xplore Press, Tunis, pp: 134-139. DOI: 10.1109/SOCPAR.2014.7007994

Savelonas, M.A., E.A. Mylona and D. Maroulis, 2012. Unsupervised 2D gel electrophoresis image segmentation based on active contours. Patt. Recog., 45: 720-731.

DOI: $10.1016 /$ j.patcog.2011.08.003
Sengar, R.S., A.K. Upadhyay, M. Singh and V.M. Gadre, 2012. Segmentation of two dimensional electrophoresis gel image using the wavelet transform and the watershed transform. Proceedings of the National Conference on Communications, Feb. 3-5, IEEE Xplore Press, Kharagpur, pp: 0-4. DOI: 10.1109/NCC.2012.6176861

Taher, R.S., N. Jamil and F.H. Yusof, 2013. Poor DNA gel electrophoresis image enhancement: Spatial Vs. frequency domain filters. Proceedings of the IEEE Conference on Systems, Process and Control, Dec. 13-15, IEEE Xplore Press, pp: 13-15. DOI: Kuala Lumpur

Talukder, S., 2011. Mathematical modelling and applications of particle swarm optimization. School of Engineering at Blekinge Institute of Technology, Blekinge.

Tandan, A. and R. Raja, 2013. A survey on particle swarm optimization methods for image segmentation. Int. J. Adv. Res. Comput. Sci. Software Eng., 3: 1539-1544.

Tillett, J. and T. Rao, 2005. Darwinian particle swarm optimization.

Wang, X.Y. and J. Bu, 2010. A fast and robust image segmentation using FCM with spatial information. Digital Signal Process., 20: 1173-1182.

DOI: $10.1016 /$ j.dsp.2009.11.007

Yang, M.S., 1993. A survey of fuzzy clustering. Math. Comput. Modell., 18: 1-16.

Yang, Y. and S. Huang, 2012. Image segmentation by fuzzy c-means clustering algorithm with a novel penalty term. Comput. Inform., 26: 17-31.

Yetirajam, M. and P.K. Jena, 2012. Enhanced color image segmentation of foreground region using particle swarm optimization. Int. J. Comput. Applic., 57: 18-23.

Zhu, Z., J. Zhou, Z. Ji and Y.H. Shi, 2011. DNA sequence compression using adaptive particle swarm optimization-based memetic algorithm. IEEE Trans. Evolut. Comput., 15: 643-658. DOI: 10.1109/TEVC.2011.2160399 